hypertension rat model, elastase activity precedes MMP activity and subsequently induces MMP activity. The matrix degradation by MMPs and elastases further interacts with growth factors such as EGF, resulting in further induction of VSMC proliferation. These data raise the question of whether targeting this pathway using elastase inhibitors will be another effective therapeutic approach. Additional investigation is needed to determine which, if any, of these abnormalities initiate PAH and which ones are the best to target for therapeutic gain. It is clear that $\mathrm{PAH}$ has a multifactorial pathobiology. As we increase our understanding of the pathways involved and their interactions, we will hopefully increase the efficacy of novel therapeutic strategies for this severe disease and further improve the palliative therapy for PAH and ultimately cure PAH.

Address correspondence to: Robyn J. Barst, New York Presbyterian Pulmonary Hypertension Center, Columbia University College of Physicians \& Surgeons, 3959 Broadway, BHN 2-255, New York, New York 10032, USA. Phone: (212) 305-4436; Fax: (212) 3421443; E-mail: rjb3@columbia.edu.
1. Humbert, M., Sitbon, O., and Simonneau, G. 2004. Treatment of pulmonary arterial hypertension. N. Engl. J. Med. 351:1425-1436.

2. Barst, R.J., et al. 1996. A comparison of continuous intravenous epoprostenol (prostacyclin) with conventional therapy in primary pulmonary hypertension. The Primary Pulmonary Hypertension Study Group. N. Engl. J. Med. 334:296-301.

3. Rubin, L.J., et al. 2002. Bosentan therapy for pulmonary arterial hypertension. N. Engl. J. Med. 346:896-903

4. Galie, N., et al. 2005. Sildenafil citrate therapy for pulmonary arterial hypertension. N. Engl. J. Med. In press.

5. Stenmark, K.R., Gerasimovskaya, E., Nemenoff, R.A., and Das, M. 2002. Hypoxic activation of adventitial fibroblasts: role in vascular remodeling. Chest. 122:326S-334S

6. Yeager, M.E., Halley, G.R., Golpon, H.A., Voelkel, N.F., and Tuder, R.M. 2001. Microsatellite instability of endothelial cell growth and apoptosis genes within plexiform lesions in primary pulmonary hypertension. Circ. Res. 88:e2-e11.

7. Balabanian, K., et al. 2002. CX (3) C chemokine fractalkine in pulmonary arterial hypertension. Am. J. Respir. Crit. Care Med. 165:1419-1425.

8. Herve, P., et al. 2001. Pathobiology of pulmonary hypertension: the role of platelets and thrombosis. Clin. Chest Med. 22:451-458.

9. Schermuly, R.T., et al. 2005. Reversal of experimental pulmonary hypertension by PDGF inhibition. J. Clin. Invest. 115:2811-2821. doi:10.1172/JCI24838.

10. Balasubramaniam, V., et al. 2003. Role of plateletderived growth factor in vascular remodeling during pulmonary hypertension in the ovine fetus. $A m$. J. Physiol. Lung Cell Mol. Physiol. 284:L826-L833.

11. Cohen, M.H., et al. 2002. Approval summary for imatinib mesylate capsules in the treatment of chronic myelogenous leukemia. Clin. Cancer Res. 8:935-942.

12. Dagher, R., et al. 2002. Approval summary: imatinib mesylate capsules in the treatment of metastatic and/or unresectable malignant gastrointestinal stromal tumors. Clin. Cancer Res. 8:3034-3038.

13. Frisdal, E., et al. 2001. Gelatinase expression in pulmonary arteries during experimental pulmonary hypertension. Eur. Respir. J. 18:838-845.

14. Lane, K.B., et al. 2000. Heterozygous germline mutations in a TGF- $\beta$ receptor, BMPR2, are the cause of familial primary pulmonary hypertension. Nat. Genet. 26:81-84.

15. Deng, Z., et al. 2000. Familial primary pulmonary hypertension (gene PPH1) is caused by mutations in the bone morphogenetic protein receptor-II gene. Am. J. Hum. Genet. 67:737-744.

16. Trembath, R., et al. 2001. Clinical and molecular genetic features of pulmonary hypertension in patients with hereditary hemorrhagic telangiectasia. N. Eng. J. Med. 345:325-334.

17. Chaouat, A., et al. 2004. Endoglin germline mutation in a patient with hereditary hemorrhagic telangiectasia and dexfenfluramine-associated pulmonary arterial hypertension. Thorax. 59:446-448.

18. Merklinger, S.L., Jones, P.L., Martinez, E.C., and Rabinovitch, M. 2005. Epidermal growth factor receptor blockade mediates smooth muscle cell apoptosis and improves survival in rats with pulmonary hypertension. Circulation. 112:423-431.

19. Cowan, K.N., Jones, P.L., and Rabinovitch, M. 2000. Elastase and matrix metalloproteinase inhibitors induce regression, and tenascin- $C$ antisense prevents progression, of vascular disease. J. Clin. Invest. 105:21-34.

20. Cowan, K.N., et al. 2000. Complete reversal of fatal pulmonary hypertension in rats by a serine elastase inhibitor. Nat. Med. 6:698-702.

\title{
Give me A5 for lipoprotein hydrolysis!
}

\section{Martin Merkel ${ }^{1}$ and Joerg Heeren ${ }^{2}$}

1Department of Internal Medicine and 2IBM II: Molecular Cell Biology, University Hospital Hamburg-Eppendorf, Hamburg, Germany.

\begin{abstract}
APOA5 is a newly identified apolipoprotein that plays a crucial role in the regulation of plasma triglyceride levels. In several human studies, common APOA5 single nucleotide polymorphisms have been strongly associated with elevated plasma triglyceride levels. In this issue of the JCI, Marçais et al. report that the rare Q139X mutation in APOA5 leads to severe hypertriglyceridemia by exerting a dominant-negative effect on the plasma lipolytic system for triglyceride-rich lipoproteins (see the related article beginning on page 2862). The presented data support the idea that the molecular mechanism of APOA5 function may include the enhancement of binding between lipoproteins and proteoglycans at the vascular wall and activation of proteoglycan-bound lipoprotein lipase.
\end{abstract}

\section{A new cause of hypertriglyceridemia}

The function of lipoproteins in vascular wall biology continues to unfold in an

Nonstandard abbreviations used: APOA5, human apolipoprotein A-V; Apoa5, murine apolipoprotein A-V; LPL, lipoprotein lipase.

Conflict of interest: The authors have declared that no conflict of interest exists.

Citation for this article: J. Clin. Invest. 115:2694-2696 (2005). doi:10.1172/JCI26712. increasingly complex story. It was more than 20 years ago that the majority of apolipoproteins known to play a major role in lipid metabolism were identified, and 9 of them (APOA1, APOA2, APOA4, APOB48, APOB100, APOC1, APOC2, APOC3, and APOE) have long been known to be most relevant to the regulation of lipoproteins. In contrast, additional apolipoproteins have only recently been identified, and their roles in lipoprotein metabolism are still being uncovered. For instance, APOM and its important function in HDL formation were just described this year (1). An important discovery in triglyceride biology was made in 2001 when a new member of the apolipoprotein family, human apolipoprotein A$\mathrm{V}$ (APOA5), was identified by postgenomic techniques. It was described independently by 2 groups; the first group used comparative sequencing (2), and the second group identified rat Apoa5 after observing the marked upregulation of the Apoa5 gene during liver regeneration (3). Since its plasma concentration is only about one-thousandth that of some other apolipoproteins (e.g., APOA1 and APOA2) (4), the relevance and function of APOA5 was not immediately fully recognized.

In this issue of the JCI, Marçais et al. describe a truncation mutation of APOA5 associated with severe familial hypertriglyc- 
A

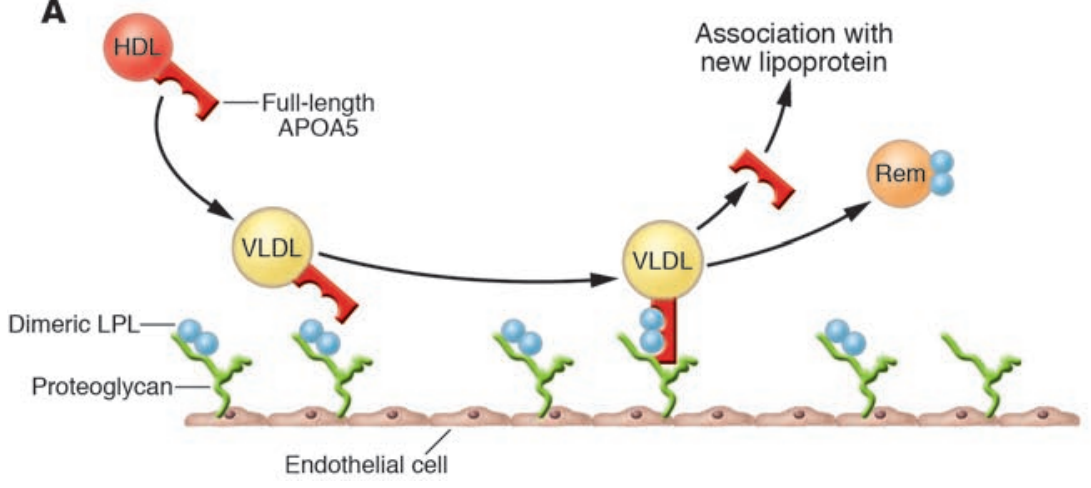

B

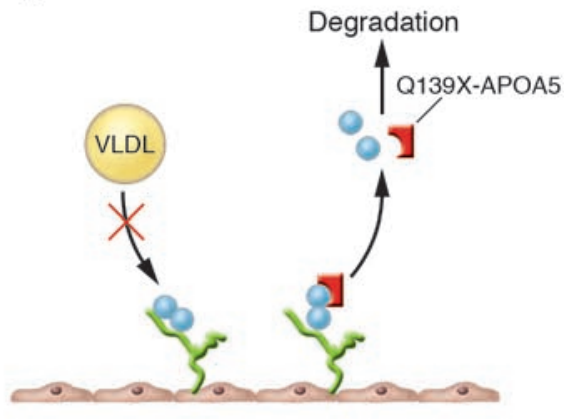

Figure 1

Triglyceride-lowering effect of APOA5. (A) Triglyceride-rich lipoproteins such as VLDL are hydrolyzed by the lipolytic action of dimeric LPL, which is bound to heparan sulfate proteoglycans on the vascular endothelium. We propose that APOA5 targets VLDL to proteoglycans, placing VLDL in close proximity to LPL. At the same time, APOA5 may activate proteoglycan-bound LPL by stabilizing the dimerized conformation or by binding to an LPL allosteric site. After hydrolysis, remnant particles (Rem) are released into the circulation, and APOA5 can be transferred and reused by other VLDL particles. In the postprandial situation, HDL can act as an APOA5 donor for intestinally derived chylomicrons. Thus, APOA5 is a potent activator of the natural lipolytic system. (B) Proposed situation in patients with the Q139X mutation in APOA5 (Q139X-APOA5). Truncated APOA5 is not associated with lipoproteins and cannot sufficiently target triglyceride-rich lipoproteins to proteoglycans. Instead, binding of the Q139X mutation in APOA5 to proteoglycan-bound LPL results in detachment and degradation of LPL.

eridemia (5). Previously, only mutations in lipoprotein lipase (LPL) (6) and APOC2 (7) have been found to cause this lipid disorder. The current study (5) consolidates the role of APOA5 as one of the key players in human triglyceride metabolism. The data presented by Marçais et al. are also consistent with the findings of several investigators showing a strong association of common APOA5 single nucleotide polymorphisms with triglyceride levels (8-12) and with familial combined hyperlipidemia $(13,14)$. In contrast to some other single nucleotide polymorphisms associated with lipid intermediate phenotypes, a surprisingly consistent picture has emerged from the literature regarding common APOA5 gene variants. But how can an apolipoprotein with a plasma concentration of only about $0.15 \mu \mathrm{g} / \mathrm{ml}$ (about $5 \mathrm{nM} / \mathrm{l}$; see ref. 4) exert such a pronounced influence on plasma triglycerides?

\section{Two theories of APOA5 function}

Since APOA5 was discovered, 2 mechanisms of APOA5 action have been proposed. One is that APOA5 may inhibit hepatic VLDL production (15-17), the other that APOA5 is an activator of intravascular triglyceride hydrolysis by LPL (18-20). Arguments can be made to support both theories; based on its structural properties (it is strongly hydrophobic), some homology with microsomal triglyceride transfer protein, and its abundant subcellular accumulation in the endoplasmic reticulum as well as the results of secretion studies performed in transfected cells, it was proposed that APOA5 may interfere with VLDL assembly (15-17). In mice, adenoviral overexpression of murine apolipoprotein A-V (Apoa5) resulted in a decreased production rate of VLDL triglycerides (20). However, these data were not confirmed by studies in Apoa5 transgenic mice (19). Most importantly, as determined by APOB kinetic studies using stable isotopes and reported in this issue of the JCI, Marçais et al. show that the VLDL production rate was normal but the fractional catabolic rate of VLDL-APOB was decreased more than 20 -fold in patients lacking normal APOA5 (5). This finding is in accordance with mouse Apoa5 overexpression data; both transgenic and adenoviral overexpression of Apoa5 resulted in a faster turnover of apoB and triglycerides from VLDL and chylomicrons (18-20).

\section{APOA5 augments plasma triglyceride hydrolysis ...}

Taking all the data together, there seems to be a strong argument for APOA5 being an activator of plasma triglyceride hydrolysis. Here is where it gets difficult; LPL is the key enzyme in intravascular triglyceride hydrolysis. Bound to endothelial proteoglycans, it acts predominantly in skeletal muscle and adipose tissue, supplying fatty acids as an energy source for these tissues. How can APOA5 at such low concentrations influence plasma hydrolysis? Usually, LPL is measured by its hydrolytic activity in the plasma after heparin injection (postheparin plasma). In the current study, Marçais et al. found lower LPL activities and mass in their APOA5-deficient patients (5). However, careful examination of mouse lines with Apoa5 deficiency or overexpression did not reveal any differences in hepatic lipase and LPL activities (19). Fruchart-Najib et al. have reported an increased hydrolysis of VLDL in vitro by free LPL in the presence of very high amounts of recombinant APOA5 $(600 \mu \mathrm{g})$ $\mathrm{ml}$; about 1,000-fold of plasma concentration) (18). Similarly, Schaap et al. showed an influence of APOA5 on LPL-mediated lipolysis of triglyceride-rich, apolipoprotein-free emulsions (20). On the other hand, using 3 different substrates, Lookene and coworkers did not detect any APOA5-mediated changes in triglyceride hydrolysis by non-proteoglycan-bound LPL (21).

\section{... only if LPL is bound to proteoglycans}

Several investigators (22-24) have postulated that plasma lipolysis is limited by factors other than the amount of postheparin plasma LPL activity. Instead, binding of triglyceride-rich lipoproteins to endothelial proteoglycans could be rate limiting. In consideration of the physiological condition in which LPL is only found bound to endothelial proteoglycans, the influence of APOA5 on LPL hydrolysis was measured in the presence and absence of proteoglycans. Without proteoglycans, APOA5 derived either from triglyceride-rich lipoproteins, 
from APOA5 transgenic mouse HDL, or from a recombinant source did not alter the LPL hydrolysis rate $(19,21)$. In the presence of proteoglycans, however, we have shown that Apoa5 led to a significant and dosedependent increase of LPL-mediated hydrolysis of VLDL and chylomicron triglycerides (19). Lookene et al. identified a proteoglycanbinding site on APOA5 (residues 186-227). Using surface plasmon resonance binding studies, they found that APOA5 could specifically increase the binding of chylomicrons and VLDL to proteoglycans. This effect was even increased by the presence of LPL and was abolished by heparin (21).

\section{How APOA5 lowers plasma triglyceride levels}

Based on these data, we propose the following model for the function of APOA5 (Figure 1). By binding to chylomicrons or VLDL at 1 site and to endothelial proteoglycans and LPL at the other, APOA5 stabilizes the endothelial lipolytic system and thereby augments LPL-mediated plasma triglyceride hydrolysis. In addition, APOA5 can stabilize dimeric LPL or, less likely, modulate the shape of triglyceride-rich lipoproteins, making them a better substrate for LPL. Direct interaction of APOA5 with LPL is in accordance with reported data (18-20). Since APOA5 can be reused and transferred to other lipoprotein particles after hydrolysis, very low APOA5 plasma concentrations are sufficient to mediate this effect. It should be mentioned that the calculation of only 1 APOA5 molecule per 1000 VLDL particles $(4,17)$ was obviously based on total APOB plasma concentration. Since APOA5 was not found bound to LDL (4) and only $6 \%$ of circulating APOB 100 is associated with VLDL (25), we estimate that about 1 APOA5 molecule is present per 24 VLDL particles. A low abundance of APOA5 is also not critical for interactions with LPL, since the amount of LPL releasable by heparin $(5 \mathrm{nM} / \mathrm{l})$ is within a similar range of plasma APOA5 level. Interactions of APOA5 with LPL could also explain the lower plasma LPL mass and activity found by Marçais et al. in their current study (5). Here, APOA5 that was truncated due to a Q139X mutation was not associated with lipoproteins and probably binds in a free form to LPL and/or proteoglycans. This seems to result in the release and subsequent degradation of LPL (Figure 1).

As described by Marçais et al., late onset hypertriglyceridemia was strictly associated with the presence of a previously described minor triglyceride-raising haplotype on the second allele (5). Although the precise mechanism of this phenomenon remains unclear, this observation is in line with a recent report by Oliva et al. describing a family with another structural mutation in the APOA5 gene (26). In this case, 5 of 10 carriers with a nonsense mutation (Q145X) in APOA5 had elevated triglyceride levels, and only the patient homozygous for this mutation developed severe hypertriglyceridemia.

Taken together, the data presented by Marçais et al. (5) clearly confirm the importance of APOA5 in the regulation of plasma triglyceride levels in humans. In addition, the concept that APOA5 directly augments lipolysis of triglyceride-rich lipoproteins at the vascular endothelium is supported. Further research needs to be done to precisely determine the molecular mechanism of APOA5 function at the endothelium, and clues for this might come from additional rare APOA5 mutations providing insight into the structure-function relationship of this fascinating apolipoprotein.

\section{Acknowledgments}

We thank Philippa Talmud for fruitful scientific discussions and for carefully reading the manuscript.

Address correspondence to: Martin Merkel, Department of Internal Medicine, University Hospital Hamburg-Eppendorf, Martinistrasse 52, 20246 Hamburg, Germany. Phone: 49-40-42803-5542; Fax: 49-40-42803-8903; E-mail: merkel@ uke.uni-hamburg.de.

1. Wolfrum, C., Poy, M.N., and Stoffel, M. 2005. Apolipoprotein $\mathrm{M}$ is required for prebeta-HDL formation and cholesterol efflux to HDL and protects against atherosclerosis. Nat. Med. 11:418-422.

2. Pennacchio, L.A., et al. 2001. An apolipoprotein influencing triglycerides in humans and mice revealed by comparative sequencing. Science. 294:169-173.

3. van der Vliet, H.N., et al. 2001. Apolipoprotein A-V: a novel apolipoprotein associated with an early phase of liver regeneration. J. Biol. Chem. 276:44512-44520.

4. O'Brien, P.J., et al. 2005. The novel apolipoprotein A5 is present in human derum, is associated with VLDL, HDL, and chylomicrons, and circulates at very low concentrations compared with other apolipoproteins. Clin. Chem. 51:351-359.

5. Marçais, C., et al. 2005. Apoa5 Q139X truncation predisposes to late-onset hyperchylomicronemia due to lipoprotein lipase impairment. J. Clin. Invest. 115:2862-2869. doi:10.1172/JCI24471.

6. Merkel, M., Eckel, R.H., and Goldberg, I.J. 2002. Lipoprotein lipase: genetics, lipid uptake and regulation. J. Lipid Res. 43:1997-2006.

7. Brunzell, J.D., and Deeb, S. 2001. Familial lipoprotein lipase deficiency, apo CII deficiency, and hepatic lipase deficiency. In The metabolic and molecular bases of inherited disease. Volume 2. C.R. Scriver, A.L. Beaudet, D. Valle, and W.S. Sly, editors. McGraw-
Hill. New York, New York, USA. 2789-2816.

8. Lee, K.W., Ayyobi, A.F., Frohlich, J.J., and Hill, J.S. 2004. APOA5 gene polymorphism modulates levels of triglyceride, HDL cholesterol and FER(HDL) but is not a risk factor for coronary artery disease. Atherosclerosis. 176:165-172.

9. Pennacchio, L.A., et al. 2002. Two independent apolipoprotein A5 haplotypes influence human plasma triglyceride levels. Hum. Mol. Genet. 11:3031-3038.

10. Talmud, P.J., et al. 2002. Relative contribution of variation within the APOC3/A4/A5 gene cluster in determining plasma triglycerides. Hum. Mol. Genet. 11:3039-3046.

11. Evans, D., Buchwald, A., and Beil, F.U. 2003. The single nucleotide polymorphism $-1131 \mathrm{~T}>\mathrm{C}$ in the apolipoprotein A5 (APOA5) gene is associated with elevated triglycerides in patients with hyperlipidemia. J. Mol. Med. 81:645-654.

12. Aouizerat, B.E., et al. 2003. Genetic analysis of a polymorphism in the human apoA-V gene: effect on plasma lipids. J. Lipid Res. 44:1167-1173.

13. Eichenbaum-Voline, S., et al. 2004. Linkage and association between distinct variants of the APOA1/C3/A4/A5 gene cluster and familial combined hyperlipidemia. Arterioscler. Thromb. Vasc. Biol. 24:167-174.

14. Mar, R., et al. 2004. Association of the APOLIPOPROTEIN A1/C3/A4/A5 gene cluster with triglyceride levels and LDL particle size in familial combined hyperlipidemia. Circ. Res. 94:993-999.

15. Weinberg, R.B., et al. 2003. Structure and interfacial properties of human apolipoprotein A-V.J. Biol. Chem. 278:34438-34444.

16. Beckstead, J.A., et al. 2003. Structure-function studies of human apolipoprotein A-V: a regulator of plasma lipid homeostasis. Biochemistry. 42:9416-9423.

17. Olofsson, S.O. 2005. ApoA-V: the regulation of a regulator of plasma triglycerides. Arterioscler. Thromb. Vasc. Biol. 25:1097-1099.

18. Fruchart-Najib, J., et al. 2004. Mechanism of triglyceride lowering in mice expressing human apolipoprotein A5. Biochem. Biophys. Res. Commun. 319:397-404.

19. Merkel, M., et al. 2005. Apolipoprotein AV accelerates plasma hydrolysis of triglyceride-rich lipoproteins by interaction with proteoglycan bound lipoprotein lipase. J. Biol. Chem. 280:21553-21560.

20. Schaap, F.G., et al. 2004. ApoAV reduces plasma triglycerides by inhibiting very low density lipoprotein-triglyceride (VLDL-TG) production and stimulating lipoprotein lipase-mediated VLDL-TG hydrolysis. J. Biol. Chem. 279:27941-27947.

21. Lookene, A., Beckstead, J.A., Nilsson, S., Olivecrona, G., and Ryan, R.O. 2005. Apolipoprotein A-V-heparin interactions: implications for plasma lipoprotein metabolism. J. Biol. Chem. 280:25383-25387.

22. Olivecrona, G., and Olivecrona, T. 1995. Triglyceride lipases and atherosclerosis. Curr. Opin. Lipidol. 6:291-305.

23. Goldberg, I.J. 1996. Lipoprotein lipase and lipolysis: central roles in lipoprotein metabolism and atherogenesis. J. Lipid Res. 37:693-707.

24. Merkel, M., et al. 2002. Inactive lipoprotein lipase (LPL) alone increases selective cholesterol ester uptake in vivo, whereas in the presence of active LPL it also increases triglyceride hydrolysis and whole particle lipoprotein uptake. J. Biol. Chem. 277:7405-7411.

25. Havel, R.J., and Kane, J.P. 2001. Structure and metabolism of plasma lipoproteins. In The metabolic and molecular bases of inherited disease. Volume 2 . C.R. Scriver, A.L. Beaudet, D. Valle, and W.S. Sly, editors. McGraw-Hill. New York, New York, USA. 2705-2716.

26. Oliva, C.P., et al. 2005. Inherited apolipoprotein A$\mathrm{V}$ deficiency in severe hypertriglyceridemia. Arterioscler. Thromb. Vasc. Biol. 25:411-417. 